[0212-7199 (2005) 22: 9; pp 441-444] ANALES DE MEDICINA INTERNA Copyright $\odot 2005$ ARAN EDICIONES, S.L.

AN MED INTERNA (Madrid) Vol. 22, N. ${ }^{\circ}$, pp. $441-444,2005$

\title{
Síndrome pneumo-renal en paciente con vasculitis de Schönlein-Henoch
}

\author{
F. J. DE LA PRADA ÁLVAREZ, A. M. PRADOS GALLARDO ${ }^{1}$, A. TUGORES \\ VÁZQUEZ, M. URIOL RIVERA, C. SAUS SARRIAS², A. MOREY MOLINA \\ Servicio de Nefrología. Hospital Universitario Son Dureta. Palma de Mallorca. ${ }^{\text {IServicio }}$ \\ de Medicina Interna. Hospital Universitario Virgen Macarena. Sevilla. ${ }^{2}$ Servicio de \\ Anatomía Patológica. Hospital Universitario Son Dureta. Palma de Mallorca
}

SCHÖNLEIN-HENOCH NEPHRITIS COMPLICATED WITH PULMO-

NARY RENAL SYNDROME

\begin{abstract}
RESUMEN
El síndrome de Schönlein-Henoch es una vasculítis sistémica que afecta a arteriolas y capilares. Aunque es un enfermedad típica de la infancia puede aparecer en edades más tardías. La enfermedad parece ser más severa en los adultos que la desarrollan. Las manifestaciones clínicas incluyen la clásica tétrada de rash cutáneo, artralgias, dolor abdominal y enfermedad renal, pero pueden verse afectados otros órganos como el miocardio, el pulmón, el uréter o el sistema nervioso central. La hemorragia pulmonar es una complicación rara del síndrome de ShönleinHenoch, que aparece generalmente en adolescentes y adultos, y está asociada con una mortalidad significativa. Presentamos a un paciente de 76 años de edad con afectación renal severa, diagnosticado por biopsia renal de síndrome de Schönlein-Henoch, que desarrolla hemorragia pulmonar tras un tratamiento inicial con bolus de esteroides por glomerulonefrítis, desapareciendo la afectación pulmonar tras un segundo ciclo de bolus de esteroides.
\end{abstract}

PALABRAS CLAVE: Síndrome de Schönlein-Henoch. Hemorragia pulmonar.

\begin{abstract}
Schönlein-Henoch purpura is a systemic vasculitic disorder involving both arterioles and capillaries. Although it is mainly a disease of early chilhood, it can occur at any age. The disease may be more severe in the few adults who develop this disorder. The clinical manifestations include a classic tetrad: rash, arthralgias, abdominal pain and renal disease. However it may affect almost every other bodily organ such us myocardium, lungs, ureter and nervous system. Pulmonary hemorrhage, a rare complication of Schönlein-Henoch purpura, has been found mainly in adolescents and adults, and it is associated with significant mortality. We present a case of 76-year-old men with Schönlein-Henoch purpura, pulmonary haemorrhage and severe renal involvement. Therapy with intravenous prednisolone resulted in resolution of the pulmonary lesions and renal failure.
\end{abstract}

KEY WORDS: Schönlein-Henoch syndrome. Pulmonary hemorrhage.

De la Prada Álvarez FJ, Prados Gallardo AM, Tugores Vázquez A, Uriol Rivera M, Saus Sarrias C, Morey Molina A. Síndrome pneumorenal en paciente con vasculitis de Schönlein-Henoch. An Med Interna (Madrid) 2005; 22: 441-444.

\section{INTRODUCCIÓN}

El síndrome de Schönlein-Henoch es una vasculítis necrotizante sistémica que afecta a vasos de pequeño calibre, y se caracteriza por el depósito tisular de inmunocomplejos que contienen IgA. Típicamente afecta a niños, y cuando afecta a pacientes de mayor edad la enfermedad suele ser más severa, con mayor afectación renal y suele requerir tratamientos más agresivos. La afectación pulmonar puede ser más frecuente de lo que clínicamente aparece ya que se ha demostrado la afectación subclínica precoz de la función pulmonar. Presentamos el caso de un paciente con síndrome neumorenal como manifestación clínica del síndrome de Schönlein-Henoch, y realizamos una valoración de los casos previamente publicados.

\section{CASO APORTADO}

Paciente de 76 años de edad, con antecedentes personales de síndrome varicoso. Ingresa en nuestro servicio por presentar hematuria macroscópica, HTA, edema facial y oliguria progresiva de una semana de evolución. En el interrogatorio confirma además la presencia de dolor abdominal y nicturia desde hace 2 meses. No infección respiratoria de vías altas previa. 
En la exploración física se objetiva un buen estado general, estando alerta y colaborador, con buena coloración de piel y mucosas. $T^{a} 36,5^{\circ}$. TA 160/95 mmHg. Se objetiva oligoanuria. No adenopatías periféricas. No soplos vasculares periféricos. Exploración cardíaca, pulmonar, abdominal y neurológica normales. Pulsos periféricos conservados. Edemas en MMII con fóvea. Síndrome varicoso en MID.

En las exploraciones complementarias destacan datos de Enfermedad renal no conocida (urea $151 \mathrm{mg} / \mathrm{dl}$, creatinina $4 \mathrm{mg} / \mathrm{dl}$ ), hipoproteinemia con hipoalbuminemia (proteínas totales 55,6 g/l, albúmina 29,4 g/l), proteinuria entre 2 y 3,6 g/24 horas, con microhematuria (eritrocitos $>200$ /campo) y cilindros granulosos en orina. El proteinograma, las inmunoglobulinas y las crioglobulinas fueron negativas. Las serologías de VHB, VHC y VIH fueron negativas. Complemento, ANA, anticuerpos antiDNA, anti-MBG y Factor reumatoide negativos. Baciloscopia y citología de esputo negativas.

El ecocardiograma fue normal. Inicialmente en la Rx de tórax sólo se observan lesiones residuales de tuberculosis en los vértices pulmonares, una posible atelectasia en base derecha y derrame pleural bilateral mínimo. La ecografía abdominal muestra un parénquima hepático homogéneo, con vesícula y vía biliar sin alteraciones. Ambos riñones son de tamaño y ecoestructura normales, sin dilatación pielocalicial.

Con el juicio clínico de Glomerulonefritis rápidamente progresiva se inicia tratamiento esteroideo, inicialmente en bolus y posteriormente vía oral, además de tratamiento sustitutivo de la función renal mediante hemodiálisis, con posterior realización de biopsia renal percutánea. En ésta se objetiva en la microscopía óptica glomérulos en los que se aprecia expansión mesangial e incremento de celularidad a expensas de polimorfonucleares neutrófilos. No se observan focos de necrosis, semilunas, ni alteraciones en la membrana basal. El intersticio muestra un aspecto en general conservado, con leve fibrosis e inflamación focal crónica. En alguno de los cortes se observa una estructura vascular correspondiente a una arteriola de mediano calibre con alteraciones en su pared PAS positivas, compatibles con necrosis fibrinoide (Fig. 1). Con técnicas de IFD se aprecia positividad intensa granular para IgA y C3 en mesangio y membrana basal.

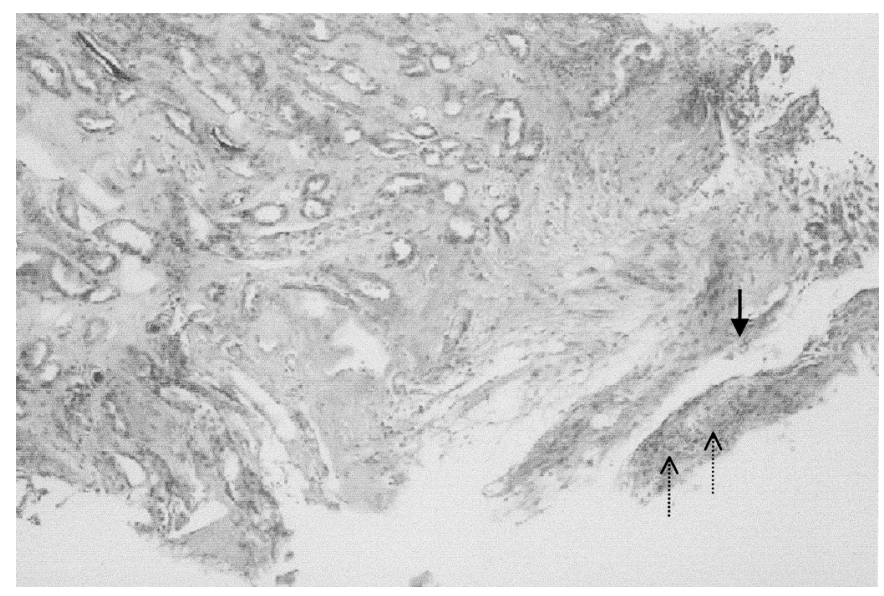

Fig. 1. Biopsia renal. Tinción PAS (x20). Estructura PAS positiva (necrosis fibrinoide en fragmento de pared de arteriola renal (flecha gruesa), con infiltrado inflamatorio transmural (flechas finas).

A los 12 días de iniciar el tratamiento el paciente presenta hemoptisis de repetición, apareciendo en la radiología convencional en el LII un infiltrado alveolar, confirmándose la sospecha de hemorragia pulmonar mediante TAC, donde aparecen áreas de ocupación alveolar a nivel de ambos lóbulos inferiores, aunque con mínima afectación derecha, compatible con hemorragia pulmonar sin nódulos pulmonares (Fig. 2). Se realizó nuevo tratamiento con bolus de esteroides con desaparición de la hemoptisis y mejoría de la imagen radiológica.

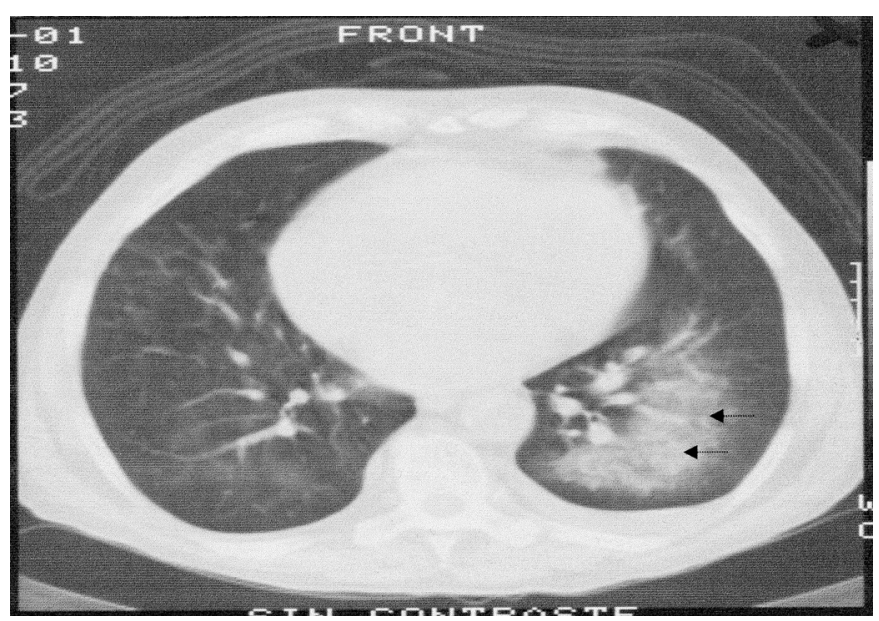

Fig. 2. TAC de tórax. Áreas de ocupación alveolar compatibles con hemorragia pulmonar (flechas).

$\mathrm{Al}$ alta presenta urea plasmática $81 \mathrm{mg} / \mathrm{dl}$ y creatinina plasmática $1,4 \mathrm{mg} / \mathrm{dl}$, con desaparición de la microhematuria. Se continuó tratamiento con esteroides orales en pauta descendente, y tres años después el enfermo permanece asintomático con función renal estable, sin hematuria y con mínima proteinuria.

\section{DISCUSIÓN}

El síndrome de Schönlein-Henoch es una vasculitis necrotizante sistémica que afecta a vasos de pequeño calibre, caracterizada por el deposito tisular de complejos inmunes que contienen $\operatorname{IgA}$. Ocurre mas frecuentemente en niños (el 50\% de los pacientes suelen tener menos de 5 años), y cuando aparece en adultos la enfermedad suele ser más severa, con mayor afectación renal y requiriendo tratamientos más agresivos (1).

Las manifestaciones clínicas clásicas en niños incluyen púrpura palpable de distribución simétrica en extremidades (100\%); artralgias (82\%); dolor abdominal de tipo cólico (63\%), pudiéndose complicar la afectación abdominal con hemorragia clínicamente evidente $(25 \%)$ o en forma de sangrado oculto $(50 \%)$, intususpección, pancreatitis, colecistitis y/o enteropatía pierdeproteinas; y enfermedad renal, que puede manifestarse como hematuria macro o microscópica, con o sin proteinuria, síndrome nefrótico, hipertensión o insuficiencia renal aguda. No obstante pueden verse involucrados otros órganos como el sistema nervioso central en forma de vasculítis cerebral, el pulmón como enfermedad intersticial o hemorragia alveolar, y puede existir afectación ocular en forma de uveítis anterior o epiesclerítis (2).

La afectación pulmonar clínica puede aparecer en un 2,4$5 \%$ de pacientes con síndrome de Schönlein-Henoch $(3,4)$, pero la afectación subclínica podría ser aún más frecuente. Se ha detectado que existe una disminución de la capacidad de difusión pulmonar del monóxido de carbono en pacientes con síndrome de Schönlein-Henoch sin síntomas ni signos de patología pulmonar, con gasometría, espirometría y hemoglobina normales, y ésta alteración revierte a la normalidad con la mejoría clínica de los pacientes. Pero la aparición de dicha alteración no se asoció con el desarrollo de afectación clínica pulmonar $(5,6)$. Esta afectación de la capacidad de difusión del monóxido de carbono se cree secundaria al deposito de 
inmunocomplejos IgA en la membrana alveolo-capilar pulmonar (6).

A nivel histológico se ha demostrado que la aparición de hemorragia pulmonar en esta enfermedad es debido a la presencia de una vasculítis leucocitoclástica de los capilares pulmonares $(7,8)$, y se ha detectado el deposito de inmunocomplejos IgA en dichos capilares (9).

También se ha descrito el desarrollo de un síndrome de Goodpasture durante el curso de una púrpura de SchönleinHenoch, con demostración de anticuerpos antimembrana basal glomerular circulantes de tipo $\operatorname{IgA}$, sugiriéndose que dado que las técnicas para determinar anticuerpos circulantes detectan principalmente anticuerpos antimembrana basal miento administrado (3). La supervivencia es de un 70,9\% (22/31 pacientes), con similar proporción de varones y mujeres (10 vs. 9 pacientes). El 50\% de éstos pacientes que sobrevivieron fueron tratados únicamente con corticoides $(11 / 22)$, y el resto tratados con corticoides e inmunosupresores, siendo azatioprina en tres pacientes, y/o ciclofosfamida en otros seis pacientes. Doce de estos veintidós pacientes eran menores de 20 años, y sólo cuatro tenía más de 30 años siendo además éstos tratados únicamente con corticoides. Entre los fallecidos, cinco varones y cuatro mujeres, hay constancia de que en cinco casos fueron tratados únicamente con corticoides, y en uno se asoció ciclofosfamida. Entre los fallecidos, cinco de los nueve casos tenían más de 45 años de edad. Por lo tanto,

\section{TABLA I}

EVOLUCIÓN, SEXO, EDAD (EN AÑOS) Y TRATAMIENTO ADMINISTRADO EN LOS CASOS PUBLICADOS DE SÍNDROME DE SCHÖNLEIN-HENOCH CON HEMORRAGIA PULMONAR

\begin{tabular}{|c|c|c|c|c|c|c|c|c|c|c|c|}
\hline \multicolumn{6}{|c|}{ Vivos } & \multicolumn{6}{|c|}{ Fallecidos } \\
\hline \multicolumn{3}{|c|}{ Varones } & \multicolumn{3}{|c|}{ Mujeres } & \multicolumn{3}{|c|}{ Varones } & \multicolumn{3}{|c|}{ Mujeres } \\
\hline Ref & Edad & Tratamiento & Ref & Edad & Tratamiento & Ref & Edad & Tratamiento & Ref & Edad & Tratamiento \\
\hline 4 & 20 & Pred & 4 & 76 & Pred & 10 & 69 & Pred & 17 & 0.5 & No \\
\hline 11 & 30 & Pred & 12 & 9 & Pred $+C$ & $4^{*}$ & 77 & Pred & $4^{*}$ & 78 & No \\
\hline 13 & 6 & Pred $+C$ & 14 & 20 & Pred & $4^{*}$ & 15 & Pred & 20 & 8 & Pred $+C$ \\
\hline 16 & 7 & Pred & 15 & 12 & Pred $+C+P l$ & $4^{*}$ & 57 & No & $4^{*}$ & 45 & Pred \\
\hline 17 & 12 & Pred $+C$ & 19 & 14 & Pred & $4^{*}$ & 8 & Pred & & & \\
\hline 18 & 15 & Pred & $4^{*}$ & 4.5 & Pred $+C$ & & & & & & \\
\hline $4^{*}$ & 16 & Pred & $4^{*}$ & 14 & Pred & & & & & & \\
\hline $4^{*}$ & 17 & Pred + Aza + C & $4^{*}$ & 53 & Pred & & & & & & \\
\hline 3 & 22 & Pred + Aza & $4^{*}$ & 10 & Pred + Aza & & & & & & \\
\hline * & 76 & Pred & & & & & & & & & \\
\hline
\end{tabular}

Pred: Prednisona; C: Ciclofosfamida; Aza: Azatioprina; PI: Plasmaféresis; ${ }^{*}$ Caso presentado; 4*: referenciado en 4.

de tipo IgG, podrían pasar desapercibidos otros casos similares (10).

Las manifestaciones clínicas de la afectación pulmonar en forma de hemorragia suelen ser palidez, disnea, taquipnea, hemoptisis y anemización progresiva. En la radiografía suelen aparecer infiltrados bilaterales u opacidades pulmonares, confirmándose estos hallazgos en la tomografía axial computerizada.

La aparición de hemorragia pulmonar en este síndrome tradicionalmente se ha asociado a alta mortalidad, y por este motivo se ha justificado un tratamiento más agresivo con fármacos inmunosupresores (11).

Se han descrito 31 casos de hemorragia alveolar en pacientes con síndrome de Schönlein-Henoch en la bibliografía (3,4,10-20), incluyendo el nuestro (Tabla I). Quince pacientes tienen sexo masculino, trece pacientes tienen sexo femenino, y en tres casos no están descritos el sexo y la edad, pero si la resolución de la hemorragia pulmonar y el trata- no parece que haya diferencias en la incidencia y en la evolución en cuanto al sexo, pero la aparición de ésta complicación en la edad joven puede ser un signo de buen pronóstico. En el tratamiento son esenciales las medidas de soporte y la medicación inmunosupresora, pero el uso exclusivo de esteroides también ha conseguido la resolución de la complicación pulmonar.

En resumen, la aparición de un síndrome neumorenal debe plantearnos la realización de un diagnóstico diferencial que incluya sus causas clásicas como el síndrome de Goodpasture, la Granulomatosis de Wegener, la Poliangítis microscópica, el Lupus Eritematoso Sistémico, y otras formas de glomerulonefritis agudas complicadas con sobrecarga de volumen y/o aumento de la permeabilidad capilar por uremia o infección pulmonar, como puede ocurrir en la glomerulonefrítis postestreptococica, pero no debemos olvidar causas menos frecuentes como la Enfermedad ateroembólica y el síndrome de Schönlein-Henoch. 


\section{Bibliografía}

1. Blanco R, Martinez-Taboada VM, Rodríguez-Valverde V, GarcíaFuentes M, González-Gay MA. Henoch-Schönlein purpura in adulthood and childhood: two different expresions of the same síndrome. Arthritis Rheum 1997; 40: 859.

2. Saulsbury, FT. Henoch-Schönlein purpura in children: Report of 100 patients and review of literature. Medicine (Baltimore) 1999; 78: 395.

3. Cream JJ, Gumpel JM, Peachey RD. Schönlein-Henoch purpura in the adult: a study of 77 adults with anaphylactoid or Schönlein-Henoch purpura. Q J Med. 1970; 39: 461-84

4. Nadrous Hassan F, Yu Aimee C, Specks Ulrich, Ryu Jay H. Pulmonary Involvement in Henoch-Schönlein Purpura. Mayo Clin Proc 2004; 79: $1151-7$.

5. Chaussain M, de Boissieu D, Califa G, et al. Impairment of lung difusion capacity in Schönlein-Henoch purpura. J Pediatr 1992; 121: 12-6.

6. Cazzato S, Bernardi F, Cinti C, Tassinari D, Canzi A, Bergamaschi R, et al. Pulmonary function abnormalities in children with Henoch-Schönlein purpura. Eur Respir J 1999; 13: 597-601

7. Weiss VF, Naidu S. Fatal pulmonary hemorrhage in Henoch-Schönlein purpura. Cutis 1979; 23: 687-8.

8. Wright WK, Krous HF, Griswold WR, Billman GF, Eichenfield LF, Lemire JM, Reznik VM. Pulmonary vasculitis with hemorrhage in anaphylactoid purpura. Pediatr Pulmonol 1994; 17: 269-71.

9. Kathuria S, Cheifec G. Fatal pulmonary in Henoch-Schönlein syndrome. Chest 1982; 82: 654-656.

10. Carreras L, Poveda R, Bas J, Mestre M, Rama I, Carrera M. Goodpasture syndrome during the course of a Schönlein-Henoch purpura. Am J Kidney Dis 2002; 39: E21.
11. Ros S, Martinez J, Gracía de Miguel MA, Olea T, de Álvaro F. Púrpura de Schönlein-Henoch de carácter familiar exteriorizada como hemorragia pulmonar. Nefrología 2004; 24: 499-502.

12. Al-Harbi NN. Henoch-Schönlein nephritis complicated with pulmonary hemorrhage but treated successfully. Pediatr Nephrol 2002; 17: 762-4.

13. Besbas N, Duzova A, Topaloglu R, Gok F, Ozaltin F, Ozen S, Bakkaloglu A. Pulmonary haemorrhage in a 6-year-old boy with HenochSchönlein purpura. Clin Rheumatol 2001; 20: 293-6.

14. Stienstra Y, Fijen JW, Tervaert JW, Dankbaar H, Zijlstra JG, van der Werf TS. [Pulmonary hemorrhage with respiratory insufficiency in HenochSchöenlein purpura. Ned Tijdschr Geneeskd 2000; 144: 617-22.

15. Paueksakon P, Hunley TE, Lee SM, Fogo AB. A 12-year-old girl with pulmonary hemorrhage, skin lesions, and hematuria. Am J Kidney Dis 1999; 33: 404-9.

16. Vats KR, Vats A, Kim Y, Dassenko D, Sinaiko AR. Henoch-Schönlein purpura and pulmonary hemorrhage: a report and literature review. Pediatr Nephrol 1999; 13: 530-4.

17. Paller AS, Kelly K, Sethi R. Pulmonary hemorrhage: an often fatal complication of Henoch-Schönlein purpura. Pediatr Dermatol 1997; 14 : 299-302.

18. Carter ER, Guevara JP, Moffitt DR. Pulmonary hemorrhage in an adolescent with Henoch-Schönlein purpura. West J Med 1996; 164: 171-3.

19. Wright WK, Krous HF, Griswold WR, Billman GF, Eichenfield LF, Lemire JM, Reznik VM. Pulmonary vasculitis with hemorrhage in anaphylactoid purpura. Pediatr Pulmonol 1994; 17: 269-71.

20. Marandian MH, Ezzati M, Behvad A, Moazzami P, Rakhchan M. Manifestations pulmonaires du purpura rheumatoïde de Schönlein-Henoch, chez un enfant de huit ans. Arch Fr Pediatr 1982; 72: 401-10. 